


$$
\begin{aligned}
& \text { QK } \\
& 5875 \\
& P_{4} \\
& \text { E24 }
\end{aligned}
$$




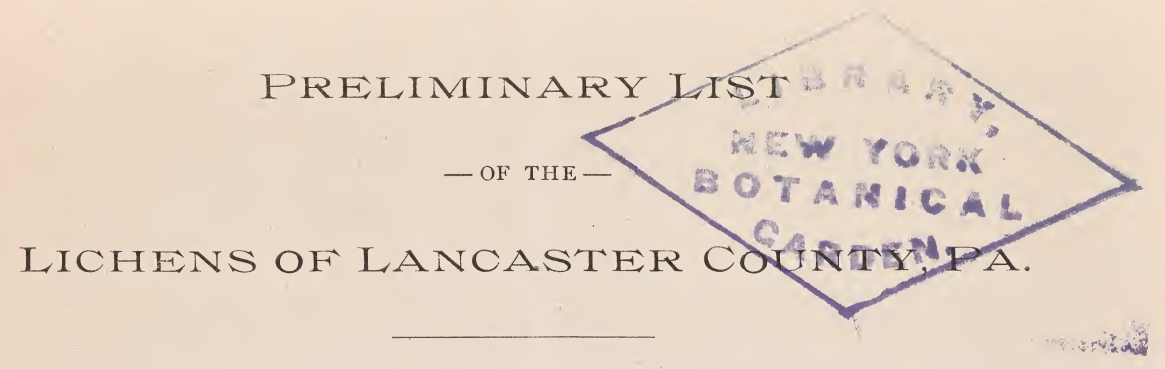

No list of the Lichens of Lancaster county has, to the best of my knowledge, yet been published.

The following is only a preliminary list of the specimens collected and analyzed as time and opportunity permitted during the years commencing in 1884 and ending in 1893 . To these I expect to add others hereafter.

The Lichens, on account of their seeming insignificance, have not received the attention given to larger plants.

The student cannot fail to find these humble plants both interesting and beautiful, and they form an important part of the economy of nature. Classed among the Cryptogamous plants, they form what may be termed the advance guard of the great repairing army of vegetation. They fill the waste places, cover the barren rocks, and dying leave their crumbling remains to feed the mosses that follow them.

An English botanist thus writes of these: "The Lichens, though generally looked upon as unworthy of notice, are of great consequence in the economy of nature. The crustaceous kinds fix upon the barest rocks, and are nourished by such slender supplies as air and the rains afford them. When these die they are converted into a very fine earth, in which the tiled Lichens find nourishment, and on their decaying and falling to dust, various mosses, as the Bryum Hypnum, etc., occupy their place. Insignificant, therefore, as these tribes may appear, they prove that means, small and simple, may accomplish the greatest ends. Lichens and mosses (especially in the colder latitudes) thus constitute the very matrix of vegetation; by successive deposits, stratum super stratum, providing beds capable of sustaining the seeds of more perfect plants (conveyed by various fortuitous circumstances) till verdure and flowers succeed to sterility, and are themselves not unfrequently crowned by the lofty pine or the knotted oak." 
$\Lambda$

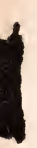




\section{PRELIMINARY LIST}

-OF THE-

LiCHENS OF LANCASTER COUNTY, PENNA.

By Mrs. Amelia F. Eby.

Usnea barbata (L.), Fr. a. florida. Tucquan Creek.

$U$. barbata, d. hirta, Fr: Mountville.

$U$. barbata, b. stringosa, Ach. Mountville.

$U$. barbata, e. plicata, Fr. Beartown.

$U$. barbata, f. dasypogon, Fr. Chestnut Hill.

$U$. longissina, Ach. Beartown.

Evernia furfuracea, Man. Cedar Hill.

E. prunastis (L.), Michx. Fite's Eddy.

Ramalina calicaris, Fr. Cedar Hill.

Cetraria glauca, Ach. Mountville.

C. cillaris, Ach. Chestnut Hill.

C. Lacunosa, Ach. Landis Valley.

C. aurescens, Tuck. Speedwell.

C. islandica (L.), Ach. Island near Fite's Eddy.

C. oakesiana, Tuck. Speedwell.

C. cucullata (Bell.), Ach. Near Fite's Eddy.

Peltigeria aphthosa, Hoffm. Chestnut Hill and Martic Forge.

P. polydactyla, Hoffm. Mountville and Safe Harbor.

P. rufescens, Hoffm. Speedwell.

P. horizontalis (L.), Hoffm. Rocks along Tucquan Creek.

P. canina, Hoffm. Chestnut Hill. 
Sticta aurata, Ach. Speedwell.

S. pulmonaria (L.), Ach. Chestnut Hill.

S. amplissima, Scop. Speedwell.

S. sylvatica, Ach. Mountville.

Parmelia caperata (L.), Ach. On rocks, Mountville.

$P$. perlata (L.), Ach. Speedwell.

P. conspersa, Ehr. Crowhill.

P. caperata (L.), Ack. On trees, Fite's Eddy.

P. perforata, Ach. Chestnut Hill.

P. crinita, Ach. Beartown.

$P$. saxatillis, Ach. Fite's Eddy.

Cladoma 'rangiferina (L.), Hoffm. Summy's Woods and Fite's Eddy.

C. rangiferina (L.), Hoffm., var. c. alpestris. Summy's Woods and Beartown.

C. rangiferina (L.), Hoffm., var. b. sylvitica. Mountville and Beartown.

C. uncialis (L.), Hoffm. Summy's Wood's.

C. squamosa, Hoffm. Chestnut Hill and Fite's Eddy.

C. cornucopioides (L.), Fr. Mountville.

C. caespiticia, Pers. Mountville.

C. pulchella, Schwein. Mountville.

C. fimbriata (L.), Fr. Crow Hill.

C. gracilis (L.), Nyl. Chestnut Hill.

C. gracilis, b. hybrida (L.), Nyl. Speedwell.

C. gracilis (L.), Nyl., var. (a.) verticilata. Speedwell.

C. curiosa (Ach.), Spreng. Beartown.

C. symphycarpa, Fr. Crow Hill.

C. mitrula, Tuck. Beartown.

C. papillaria (Ehr.), Hoffm. Beartown.

C. fimbriata, b. tubaformis (L.), Fr. Crow Hill.

C. Boryii, Tuck. Summy's Woods.

C. furcata, Huds., var. b. racemosa. Chestnut Hill.

C. furcata, Huds. Chestnut Hill.

C. pyxidata, Fr. Mountville.

C. cristatella, Tuck. Little Conestoga, Miss M. Zahm.

Bacomyces roseus, Pers. Chestnut Hill.

B. aerugrnosus (Scop.), D. C. Crow Hill.

Biatora vernalis (L.), Fr. Chestnut Hill. 
Umbilicaria Dillenii, Tuck. Rocks along Hammer Creek.

U. pustulata (L.), Hoffm. b. papulosa. Chestnut Hill.

$U$. Muhlenbergii, Tuck. Speedwell.

Endocarpon miniatum var. Muhlenbergii, Ach. Conestoga Creek.

E. miniatum, Ach., var. complinatum. Conestoga creek.

Stringula complanata (Fr.), Mont. Nyl. Smithville.

Physica stellaris, L. Mountville.

P. hypoleuca (L.), Michx. Fite's Eddy.

$P$. leucomela (L.), Michx. Fite's Eddy.

P. obscura (Ehr.), Nyl. Hershey's dam.

Lecanora palida (Schreb.), Schaer. Pequea.

L. elatina (L.), Ach. Glen Manor.

L. subfusca (L.), Ach. Glen Manor.

Theloschistes parietinus (L.), Norm. Conewago.

Conotrema urceolatum (Ach.), Tuck. Glen Manor.

Stereocaulon paschale (L.), Fr. Island above Peach Bottom.

S. condensatum, Hoffm. Beartown.

Anthonia spectabilis, Fr. Habecker's woods.

Graphis scripta, L. Fite's Eddy.

Note: In naming some of the most difficult specimens in the above list, Mrs. Eby acknowledges the assistance of Clara E. Cummings, of Wellesley College, Mass. 


